RA. PI3Kdelta isoform expression was recently identified as a key regulator of FLS growth and survival, suggesting that it could contribute to synoviocyte aggressive behaviour. Therefore, we assessed the role of PI3Kdelta in synoviocyte invasion and matrix degradation using isoform selective PI3K inhibitors.

Methods FLS were cultured in Matrigel coated transwells. PI3K inhibitors or vehicle were added to the upper chamber and PDGF was used as a chemoattractant in the lower chamber. The invading cells were quantified by staining the philtres with $1 \%$ crystal violet. F-actin was visualised with Rhodamin phalloidin and analysed with fluorescent microscopy. Rac1 activation was measured using PAK1/ PBD GST pull down and quantified by Western blot analysis. PI3K inhibitors: pan (GDC-0941), PI3Kalpha (A66), PI3Kbeta (TGX-221), PI3Kgamma (AS-252424), PI3Kdelta (INK007 and CAL-101) and PI3K-delta/gamma (IPI-145).

Results PDGF-directed invasion was completely inhibited by the pan PI3K inhibitor ( $1 \mathrm{uM})$. To define the role of the individual isoforms, we tested the effect of the isoform selective PI3K inhibitors. PI3Kdelta inhibition (INK007) significantly decreased the number of invading cells, with $60 \pm 5 \%$ inhibition at $1 \mathrm{uM}(\mathrm{p}<0.04)$. Similar results were observed with two other inhibitors with distinct chemical structures (CAL-101 and INK055). The PI3Kalpha inhibitor decreased invasion by $40 \pm 5 \%$ while PI3Kbeta and PI3Kgamma inhibitors had no effect. Phalloidin staining was then used to visualise FLS actin rearrangement in response to PDGF with or without PI3K inhibitors. PI3Kdelta inhibition by INK007, CAL-101 and IPI-145 decreased lamellipodia formation by $50 \pm 6 \%(p<0.05)$. Similar inhibition was seen with the pan PI3K inhibitor, while the selective inhibitors of PI3Kalpha, PI3Kbeta or PI3Kgamma had no effect. We then hypothesised that PI3Kdelta might modulate activation of Rho GTPases in synoviocytes, which regulate actin organisation. PI3Kdelta inhibition with INK007 had no effect on baseline Rac1 activation but blocked activation in response to PDGF by $95 \pm 6 \%$ $(p<0.03)$. Similar findings were observed with the pan PI3K inhibitor, while PI3Kalpha inhibition had no significant effect.

Conclusions PI3Kdelta is a major regulator of FLS migration and invasion and functions by inhibiting Rac1 activation and modulating $\mathrm{F}$ actin cytoskeleton rearrangement. These observations, together with previous findings that PI3Kdelta regulates FLS growth and survival, suggest that PI3Kdelta inhibition could be chondroprotective in RA by modulating synoviocyte growth, migration and invasion.

\section{A9.3 DELETION OF mPGES-1 AFFECTS FATTY ACID COMPOSITION AND EICOSANOID PROFILES IN MICE}

doi:10.1136/annrheumdis-2013-203223.3

\begin{abstract}
1,2Helena Idborg, ${ }^{3}$ Petter Olsson, 'Patrick Leclerc, ' 'Joan Raouf, 'Per-Johan Jakobsson, 1,2Marina Korotkova. 'Rheumatology Unit, Department of Medicine, Karolinska Institutet, Sweden; ${ }^{2}$ Actar AB, Solna, Sweden; ${ }^{3}$ Department of Analytical Chemistry, Stockholm University, Sweden
\end{abstract}

Background mPGES-1 is considered an attractive alternative target for anti-inflammatory treatment with improved selectivity and safety compared to NSAIDs. Genetic deletion or pharmacological inhibition of mPGES-1 activity or expression down-regulate inflammation and pain in experimental models of arthritis. However, a detailed understanding of the molecular mechanisms and pathways affected by deletion/inhibition of mPGES-1 is essential before mPGES-1 inhibitors can safely be applied in the clinics.

Objectives To investigate the effect of mPGES-1 deletion on the levels of bioactive lipid mediators such as the eicosanoids (downstream cascade) and fatty acids (up-stream cascade).

Methods Peritoneal macrophages (PM) from wild type (WT) and knock-out (mPGES-1\%, KO) mice were induced with LPS for $16 \mathrm{~h}$. Cells were harvested for gene expression analysis and supernatants were collected for eicosanoid analysis. Gene expression profiling in
WT and $\mathrm{KO}$ macrophages was performed using the microarray analysis (Applied Biosystems). Eicosanoid profiling of approximately 30 compounds was performed using LC-MS/MS. Fatty acid composition of total lipids in spleen and brain homogenates of WT and $\mathrm{KO}$ mice were determined using GC/FID.

Results Microarray analysis revealed that genetic deletion of mPGES-1 affected expression of genes related to lipid metabolism and mainly associated with eicosanoid, fatty acid and phospholipid metabolism, (e.g., Pla1A, Ptgis, Fabp3, Cept1, Comt etc). Compared to WT, mPGES-1 deficient PM displayed a markedly attenuated increase in $\mathrm{PGE}_{2}$ production upon LPS stimulation, and exhibited significantly increased levels of $\mathrm{PGD}_{2}$ metabolites such as 15 -deoxy- $\Delta^{12,14}$ PGJ ${ }_{2}$ and 15 -deoxy- $\Delta^{12,14} \mathrm{PGD}_{2}(\mathrm{p}<0.05)$. There were significant differences in the fatty acid composition in spleen (e.g., palmitoleic acid decreased in $\mathrm{KO}, \mathrm{p}<0.05$ ) and brain (e.g., myristic acid decreased in $\mathrm{KO}, \mathrm{p}<0.05$ ) of $\mathrm{KO}$ and WT mice, suggesting that feed-back mechanisms are alternated by the deletion of m-PGES-1.

Conclusions Data reveals that mPGES-1 deficient PMs displayed shunting towards PGD2 pathway, i.e., towards anti-inflammatory metabolites, upon LPS stimulation compared to WT PMs. Moreover, mPGES-1 depletion alters the fatty acid composition of tissue lipids.

These effects of inducible $\mathrm{PGE}_{2}$ on lipid metabolism have important implications for future mPGES-1 inhibitors and deserve further investigation.

\section{A9.4 FLT3L-DEPENDENT CD103 + DC ARE CRUCIAL FOR THE INITIATION AND MAINTENANCE OF COLLAGEN-INDUCED ARTHRITIS}

doi:10.1136/annrheumdis-2013-203223.4

1,2M Inês Ramos, 1,2Saida Aarrass, ${ }^{3}$ SE Jacobsen, 'Paul P Tak, 1,2M Cristina Lebre. ${ }^{1}$ Departmtment of Clinical Immunology and Rheumatology; ${ }^{2}$ Department of Experimental Immunology, Academic Medical Center/University of Amsterdam, Amsterdam, The Netherlands; ${ }^{3}$ Stem Cell Biology Laboratory, University of Oxford, UK

Background and Objectives Dendritic cells (DCs) are a heterogeneous population of antigen-sensing and-presenting cells that play an important role for the initiation of protective and pathogenic immunity. Flt3L is a growth factor that can drive DC development from bone marrow (BM) progenitors and it is crucial for steadystate DC maintenance. Collagen-induced arthritis (CIA) induction requires dermal DC uptake, processing, migration and presentation of antigen to lymph node (LN) T cells. The objective of this study was to find the mechanism of protection in CIA observed in mice lacking Flt3L (Flt3L $\mathrm{L}^{-/}$).

Materials and Methods CIA was induced in Flt3L $\mathrm{L}^{-1}$ and WT littermates. The severity of the arthritis was assessed using an established semiquantitative scoring system (0-4). In vitro uptake and migration was assessed by using BM-DC (differentiated with GM-CSF for9days). OVA-FITCwasinjectedintradermallyin Flt3L $\mathrm{L}^{-1}$ and WT animals, after $36 \mathrm{~h}$ LNs were collected and the number of FITC positive cells was determined. Antigen presentation was studied by adoptive transfer of CFSE-labelled OT-I or OT-II T cells + OVA (i.d.) into Flt $3 \mathrm{~L}^{-/}$and WT animals. After 3 days LNs were collected and $\mathrm{T}$ cell proliferation was analysed by CFSE dilution.

Results $\mathrm{Flt} 3 \mathrm{~L}^{-/}$mice (steady-state) have severe reductions in DC populations in lymphoid and non-lymphoid organs. Flt3 $3 \mathrm{~L}^{-1-}$ mice are protected from CIA showing impaired $\mathrm{T}$ and $\mathrm{B}$ cell responses. Flt $3 \mathrm{~L}^{-1}$ mice have reduced dermal CD11b + DCs and CD103+ DCs are almost absent. In addition, the amount of DCs carrying antigen reaching the $\mathrm{LN}$ in $\mathrm{Flt} 3 \mathrm{~L}^{-1}$ mice was reduced compared with WT. In vitro and in vivo experiments using BMDCs demonstrated that Flt $3 \mathrm{~L}^{-}$BMDCs have the same capacity to uptake and migrate as WT BMDCs. Moreover, adoptive transfer of OT-I and OT-II T cells + OVA in Flt3 $\mathrm{L}^{-/}$mice resulted in a dramatic reduction of total cell 
proliferation and more importantly less divisions compared with WT animals. FACS analysis of WT and Flt3L $1-$ mice synovium at the acute phase of CIA showed that CD11b+ DC are present and increased in arthritic animals compared with immunised but not arthritic animals. CD103+ DC are only present in WT animals, and increased in animals with a high clinical score.

Conclusions Our data shows that antigen presentation in Flt $3 \mathrm{~L}^{-1}$ mice is impaired. As CD103+ DC are important in presenting and cross-presenting antigens our data reveals an important role for CD103 + DC in both induction and maintenance of CIA. Specifically targeting CD103+ DC could provide a novel antirheumatic strategy.

\section{A9.5 IDENTIFICATION AND VALIDATION OF A PROTEIN COMBINATION INCLUDING S100A9 ABLE TO PREDICT THE RESPONSE TO THE MTX/ETANERCEPT ASSOCIATION IN RHEUMATOID ARTHRITIS PATIENTS}

doi:10.1136/annrheumdis-2013-203223.5

${ }^{1,2} \mathrm{~A}$ Obry, 'T Lequerré, ${ }^{2} \mathrm{~J}$ Hardouin, ${ }^{1,3,4} \mathrm{O}$ Boyer, ${ }^{1,4} \mathrm{O}$ Vittecoq, ${ }^{2 \mathrm{P}}$ Cosette. ${ }^{1}$ INSERM 905, Institute for Biomedical Research, University of Rouen, Rouen; ${ }^{2}$ UMR 6270 CNRS,

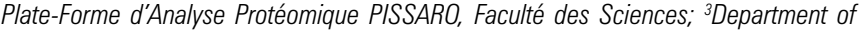
Immunology, Rouen University Hospital; ${ }^{4}$ Department of rheumatology, Rouen University Hospital

Background The number of biologic agents in Rheumatoid Arthritis (RA) is continuously increasing. However, clinicians observe that around 30 to $40 \%$ of treated patients fail to respond to TNF $\alpha$ blocking agents. One way to optimise the drug prescription is to identify predictive markers of drug responsiveness.

Objectives To identify a combination of serum proteins whose expression profile would predict the RA patients responses to the association of methotrexate (MTX) and etanercept (ETA) by mass spectrometry-based quantification methods and ELISA.

Methods A "cohort discovery phase" of 23 patients with active RA was treated by a subcutaneous injection of. The clinical efficacy of these drugs was evaluated with the DAS28 score after 6 months of treatment according to the EULAR response criteria. For proteomic analysis, a serum sample was collected in patients prior to treatment exposure. A "label free" approach on the whole proteome was performed by mass spectrometry on the 25 sera. Accordingly, the proteome of each sample was extracted and in-gel digested. The resulting peptides were analysed by LTO Orbitrap ${ }^{\circledR}$ (ThermoFisher). Differential analysis between responder and non responder samples was performed with LCMS ProGenesis ${ }^{\circledR}$ (Nonlinear Dynamics). To validate these results a relative quantification of selected protein was performed on the second "cohort validation phase" by ELISA. The proteome of peripheral blood mononuclear cells (PBMC) from a second cohort of seven patients with similar characteristics has also been studied by the same label free approach.

Results The label free approach revealed 12 differentially expressed serum proteins according to patient response. This combination of proteins was used to build a Random Forest statistical model to predict the patient's status. This model was validated by a blind test on a panel of seven patients. Moreover, these results have shown the protein S100A9 overexpression in both the serum and the PBMCs from responder's patients and this expression was confirmed by ELISA.

Conclusions The label free approach has identified a combination of predictive markers of response to MTX treatment/ETA. Thus, using sera samples collected in patients prior to treatment exposure, it is possible to predict response to treatment with a small error. These proteins represent interesting candidate biomarkers of response that must be validated in a larger population. Already identified as a diagnostic and prognostic biomarker of RA, the S100A9 protein has been identified as a predictive biomarker of response both in serum and in PBMCs.

\section{A9.6 IDENTIFICATION OF NEW POTENTIAL THERAPEUTIC TARGETS FOR THE TREATMENT OF RHEUMATOID ARTHRITIS: ENTPD1 (CD39) AND 5NTE1 (CD73)}

doi:10.1136/annrheumdis-2013-203223.6

1,2Jonathan D Finn, ${ }^{2}$ Lisa van Baarsen, ${ }^{1,2}$ Jan van Ittersum, ${ }^{1,2}$ Ciska Braam, ${ }^{2}$ Maria C Lebre, 1,2Paul P Tak, 1,2Margriet J Vervoordeldonk. 'Arthrogen BV, Amsterdam, The Netherlands; 'Div. of Clinical Immunology and Rheumatology, Academic Medical Center/University of Amsterdam, Amsterdam, The Netherlands

Background and Objectives Adenosine and ATP are known to have important immunomodulatory properties. Extracellular ATP has multiple roles in inflammation and can act as a damageassociated molecular pattern (DAMP) that can activate the immune system. Conversely, adenosine is primarily anti-inflammatory and can inhibit the production of pro-inflammatory molecules by immune cells. The modulation of ATP and adenosine levels are an essential part of the induction and resolution of an inflammatory response. ENTPD1 (CD39) is a membrane-bound ectonucleoside triphosphate diphosphohydrolase enzyme that converts ATP and ADP to AMP 5NTE1 (CD73) is a 5' ecto-nucleotidase that dephosphorylates AMP to form adenosine. We investigated the role of genes in the adenosine pathway in patients with rheumatoid arthritis (RA) and determined whether expression of CD39 and CD73 would have an effect in an in vitro inflammation model.

Materials and Methods Gene expression analysis using 43k cDNA microarrays (Stanford Functional Genomics Facility) was performed on total RNA extracted from RA synovial tissues obtained by arthroscopy. Adenosine pathway gene expression was compared between high-inflammation versus low-inflammation tissue type synovial biopsies. ATPase levels were measured in synovial fluid (SF) from RA $(n=10)$ or osteoarthritis $(O A)(n=6)$ patients. Adeno-associated viral (AAV) vectors expressing CD39 or CD73 were generated and used to transduce HEK 293 cells or RA fibroblast-like synoviocytes (FLS) and these transduced cells were co-cultured with LPS-activated human monocytes (THP-1) in the presence of ATP. Pro-inflammatory cytokine/chemokine (IL-6, CCL2) production was measured by ELISA.

Results Genes involved in the ATP:adenosine pathway, including CD73, were differentially expressed in high-inflammation synovial tissues, consistent with the hypothesis that there is skewing of the ATP:adenosine balance during inflammation. The half-life of ATP was significantly increased in SF from RA patients compared with $\mathrm{OA}\left(\mathrm{t}_{1 / 2}=8.0\right.$ versus $4.5 \mathrm{~min}$., $\left.\mathrm{p}=<0.05\right)$, indicating that there was a significant decrease in ATPase activity in RA SF HEK 293 cells and RA FLS cell lines transduced with CD39-and/or CD73-expressing AAV5 vectors demonstrated high CD39 and CD73 activity. THP-1 cells stimulated with LPS showed lower levels $(>80 \%$ reduction $p=<0.05)$ of IL- 6 and CCL 2 secretion when co-cultured with CD39 and CD73 expressing HEK293 cells or FLS cells in the presence of ATP.

Conclusions Together, these data suggest that synovial inflammation in RA is characterised by skewing of the ATP:adenosine balance. This could be reversed by overexpression of CD39 or CD73. Thus, these data show that the ATP:adenosine pathway may be a novel therapeutic target for the treatment of RA.

\section{A9.7 LOSS OF PTEN IN MYELOID CELLS CONTROLS INFLAMMATORY BONE DESTRUCTION BY REGULATING THE OSTEOCLASTOGENIC POTENTIAL OF MYELOID CELLS}

doi:10.1136/annrheumdis-2013-203223.7

'Stephan Blüml, ${ }^{2}$ Gernot Schabbauer, ${ }^{2}$ Martin Friedrich, 'Antonia Puchner, 'Victoria Saferding, ${ }^{2} E m i n e$ Sahin, ${ }^{2} T o b i a s$ Lohmeyer, 'Birgit Niederreiter, 'Josef Smolen, ${ }^{1}$ Kurt Redlich. 'Division of Rheumatology, Internal Medicine III, Medical University of Vienna, Austria; ${ }^{2}$ nstitute for Vascular Biology and Thrombosis Research, Center for Biomolecular Medicine and Pharmacology, Medical University Vienna, A-1090 Vienna, Austria; 3 Institute of Immunology, Biomedical Sciences Research Center (BSRC) "Alexander Fleming," Vari, Greece 